cells from RA synovial biopsy cell suspensions $(n=4)$ showed surface expression of CD28.

Conclusions: We have generated a unique dataset from different phases of RA progression. Our results provide guidance for the selection of co-signaling molecules as therapeutic targets as well as for preventing the progression to RA. In particular, $C D 28$ expression was elevated in synovial tissue biopsies before the development of RA and it was also expressed on the majority of synovial tissue infiltrating CD4+ and CD8+ T cells from RA patients. These observations provide rationale to target $C D 28$ for both $\mathrm{RA}$ treatment and disease interception.

Disclosure of Interest: A. Walsh Shareholder of: Johnson \& Johnson, Employee of: Johnson \& Johnson, M. Canavan: None declared, Y. Guo Shareholder of: Johnson \& Johnson, Employee of: Johnson \& Johnson, T. McGarry: None declared, X. Yin Shareholder of: Johnson \& Johnson, Employee of: Johnson \& Johnson, M. Wechalekar Grant/research support from: Johnson \& Johnson, M. Smith Consultant for: Johnson \& Johnson, S. Proudman: None declared, C. Orr: None declared, S. Kelly: None declared, C. Pitzalis Grant/research support from: Johnson \& Johnson, Consultant for: Abbott, Astellas, Medlmmune, BMS, Celgene, Grunenthal, GSK, MSD, Pfizer, Sanofi, Roche, UCB, D. Veale: None declared, U. Fearon: None declared, S. Nagpal Shareholder of: Johnson \& Johnson, Employee of: Johnson \& Johnson

DOI: 10.1136/annrheumdis-2017-eular.1343

\section{THU0012 HLA-DBR1 ALLELES PROFILE IN PATIENTS WITH RHEUMATOID ARTHRITIS: RELATION TO DISEASE SUSCEPTIBILITY AND SEVERITY}

A. Paradowska-Gorycka ${ }^{1}$, B. Stypinska ${ }^{1}$, E. Haladyj $^{2}$, K. Romanowska-Prochnicka ${ }^{2}$, A. Pawlik ${ }^{3}$, M. Olesinska ${ }^{2} .{ }^{1}$ Department of Molecular Biology; ${ }^{2}$ Department of Connective Tissue Diseases, National Institute of Geriatrics, Rheumatology and Rehabilitation, Warsaw; ${ }^{3}$ Department of Physiology, Pomeranian Medical University, Szczecin, Poland

Background: Rheumatoid arthritis (RA) is a complex, chronic autoimmune disease (AID) that affects approximately $0.5 \%-1 \%$ of the population worldwide. $\mathrm{RA}$ is considered to be complex diseases of unknown etiology, where the environmental, immunological and genetic factors contributed to susceptibility and severity of the disease. Since the first evidence suggesting the involvement of human leukocyte antigens (HLAs) in RA was reported in 1969, the HLA region have been at the center of genetic studies of RA.

Objectives: We undertook this study to elucidate the association between HLA-DRB1 alleles profile and susceptibility to and severity of RA in a Polish population.

Methods: We analyzed the data on a total of 472 RA patients in whom HLADRB1 allele genotyping had been performed. DNA sequences for exon 2 of the DRB1 locus were typed using polymerase chain reaction-sequence-based typing (PCR-SBT)

Results: Identification of the variants potentially associated with risk and protection was carried out by comparison to the DKMS Polish Bone Marrow Donor Registry (41306 alleles). A significant increase in the frequency of the HLA-DRB1*15:01 $(p=0.001), D R B 1{ }^{*} 04(p<0.0001), D R B 1{ }^{*} 01: 01(p<0.0001), D R B 1 * 10: 01(p=0.003)$ and ${ }^{*} 09: 01 \quad(p<0.0001)$ were identified in patients with RA and showed strong association with the disease susceptibility. Furthermore, the HLA-DRB1*07:01 allele was found to be protective $(p<0.0001)$ in our RA patients. In the RA patients with HLA-DRB1*52:01 allele the mean value of PLT $(p=0.03)$ and organ symptoms $(p=0.02)$ were more frequent than in RA patients without this allele. In RA patients with $\mathrm{DRB}^{*} 01: 01, \mathrm{DRB}^{*} 04$ as well as $\mathrm{DRB} 1{ }^{*} 15: 01$ alleles the number of tender joints $(p=0.03)$, diabetes $(p=0.03)$ and renal failure) $p=0.03$ ) were observed less frequently compared with RA patients without these alleles. We also observed that in RA patients with DRB1*10:01 the VAS score was higher than in RA patients without this allele.

No association was detect between the HLA-DRB1*07:01 and DRB1*09:01 and disease activity and laboratory parameters among RA patients.

Conclusions: Current finding indicated that HLA-DRB1*15:01 DRB1*04, DRB1*01:01 and DRB1*10:01 alleles may be associated with susceptibility to as well as severity of RA.

Disclosure of Interest: None declared

DOI: 10.1136/annrheumdis-2017-eular.5794

\section{THU0013 TOWARDS THE DEFINITION OF A RISK MODEL PROFILE OF PERICARDITIS IN SYSTEMIC LUPUS ERYTHEMATOSUS: A GENETIC STUDY}

C. Perricone ${ }^{1}$, F. Ceccarelli ${ }^{1}$, C. Ciccacci ${ }^{2}$, S. Rufini ${ }^{2}$, C. Alessandri ${ }^{1}$ F.R. Spinelli ${ }^{1}$, E. Cipriano ${ }^{1}$, C. Politi ${ }^{2}$, A. Latini ${ }^{2}$, G. Novelli ${ }^{2}$, G. Valesini ${ }^{2}$, P. Borgiani ${ }^{2}$, F. Conti ${ }^{1}$. ${ }^{1}$ Lupus Clinic, Rheumatology, Department of Internal Medicine, Sapienza University of Rome; ${ }^{2}$ Department of Biomedicine and Prevention, University of Rome Tor Vergata, Rome, Italy

Background: We have recently demonstrated that polymorphisms (SNPs) in TRAF3IP2 gene are associated with susceptibility for systemic lupus erythematosus (SLE) and can predispose to the development of pericarditis (1). Moreover, we found that other genes previously associated with susceptibility to SLE (MIR1279, STAT4, PTPN2) seem to be involved in the development of pericarditis (2). At this purpose, we built a genetic risk model for the development of pericarditis in SLE (3).

Objectives: We aimed to expand the knowledge on the genetic risk of pericarditis in SLE by studying the role of rs2205960 (TNFSF4) and rs2233945 (ATG16L1) SNPS, previously associated with SLE susceptibility, improving our genetic risk model.

Methods: We recruited SLE patients (diagnosed according to 1997 revised ACR criteria) and healthy subjects served as controls. Study protocol included complete physical examination and blood drawing. The clinical and laboratory data were collected in a standardized, computerized, electronically-filled form (3). Clinical and laboratory features were assessed with a dichotomous score (present $=1$; absent=0). SNPs genotyping was performed by allelic discrimination assay. A case/control association study and a genotype/phenotype correlation analysis were performed and a risk profile model for pericarditis in SLE was built. Results: Three-hundred fifteen SLE patients [285 F (90.5\%), $30 \mathrm{M}(9.5 \%)$, mean age $43.11 \pm 11.28$ years, mean age at onset $32.19 \pm 11.84$ years] and 278 healthy controls were enrolled. Pericarditis was present in 56 (17.8\%) SLE patients. Deviations from Hardy-Weinberg equilibrium for the studied SNPs were not observed. The variant alleles of the rs2205960 (TNFSF4, $\mathrm{P}=0.013, \mathrm{OR}=2.14$ ) and of the rs2233945 (ATG16L1 $P=0.009, O R=2.32$ ) were significantly associated with susceptibility to pericarditis. A risk profile model for pericarditis considering the risk alleles of TRAF3IP2, MIR1279, STAT4, PTPN2, TNFSF4 and ATG16L1 showed that patients with more than 5 risk alleles have a significantly higher risk to develop pericarditis $(\mathrm{P}<0.001, \mathrm{OR}=8.01)$. Anti-Sm antibodies were the only laboratory parameter associated with the development of pericarditis. Thus, a multivariate analysis by binary regression analysis, considering as dependent variable the presence or absence of pericarditis and as independent variables all the studied SNPs associated with pericarditis, was performed. In a stepwise approach including anti-Sm, TRAF3IP2, MIR1279, STAT4, PTPN2, TNFSF4 and ATG16L1 SNPs, the model explains about $25 \%$ ( $R^{2}$ Cox \&Snell) of the variability involved in the susceptibility to pericarditis.

Conclusions: We describe for the first time the contribution of TNFSF4 and ATG16L1 SNPs in pericarditis development in SLE patients. We improved our genetic risk profile model to better and earlier identify SLE patients more susceptible to develop this complication.

References:

[1] Perricone C, Ciccacci C, et al. Immunogenetics. 2013 Oct;65(10):703-9.

[2] Ciccacci C, Perricone C, et al. PLoS One. 2014 Nov 4:9(11):e111991.

[3] Ciccacci C, Perricone C, et al. Lupus. 2016. doi: 10.1177/0961203316679528. Disclosure of Interest: None declared

DOI: 10.1136/annrheumdis-2017-eular.6028

\section{THU0014 NO ASSOCIATION OF SNP RS3761847 WITH THE EXPRESSION OF THE TRAF1-C5 LOCUS AND INVASION OF RHEUMATOID ARTHRITIS SYNOVIAL FIBROBLASTS}

D. Ferry ${ }^{1,2}$, M. Trenkmann ${ }^{2}$, K. Creevey ${ }^{2}$, A.G. Wilson ${ }^{2} .{ }^{1}$ School of Medicine; ${ }^{2}$ The Conway Institute of Biomolecular and Biomedical Research, University College Dublin, Dublin, Ireland

Background: Polymorphisms in the TRAF1-C5 locus have been associated with susceptibility to rheumatoid arthritis (RA). A single nucleotide polymorphism (SNP) rs3761847, located in the first intron of the TRAF1 gene, has been identified as an RA risk locus in a genome-wide association study (GWAS); the relationship of rs3761847 with joint damage are unclear. One of the key mechanisms of joint destruction is the degradation of and invasion of cartilage by RA synovial fibroblasts (RASF).

Objectives: To determine whether the expression of TRAF1-C5 genes and the invasion of RASF are associated with the genotype at rs3761847.

Methods: The genotype at rs3761847 was determined using TaqMan Allelic Discrimination Assay. Invasion assays were performed using Matrigel-coated invasion chambers. Peripheral blood mononuclear cells (PBMC) were obtained by density gradient centrifugation. Neutrophils were isolated from the red blood cell layer by erythrocyte lysis; $\mathrm{CD}_{14}{ }^{+}$cells were separated using magnetic cell sorting. Macrophages were differentiated from plastic-adhered monocytes in M-CSF $(50 \mathrm{ng} / \mathrm{ml})$ for 8 days. Cells were stimulated with lipopolysaccharide (LPS, $100 \mathrm{ng} / \mathrm{ml})$ plus interferon $\gamma(\mathrm{IFN} \gamma, 20 \mathrm{ng} / \mathrm{ml})$, interleukin $4(\mathrm{IL}-4,20 \mathrm{ng} / \mathrm{ml})$ or tumour necrosis factor $\alpha(\mathrm{TNF}-\alpha, 10 \mathrm{ng} / \mathrm{ml})$. Gene expression was measured by SYBR Green real-time PCR using HPRT1 as a housekeeping gene.

Results: rs3761847 was in Harvey-Weinberg equilibrium in RASF $(n=54, p=0.98$ ) with genotype frequencies of $0.42 \mathrm{AA}, 0.45 \mathrm{AG}$ and $0.13 \mathrm{GG}$. When grouped by genotype, the expression of TRAF1 (total), TRAF1 transcript variant 2 and complement C5 mRNA showed no significant difference $(p=0.929, p=0.583$, $p=0.980$ respectively). This was also the case for invasion of RASF ( $n=43$, $\mathrm{p}=0.548$; mean cell count $10.4 \mathrm{AA}, 8.3 \mathrm{AG}$ and $11.1 \mathrm{GG})$. There was no correlation of genotype with common RA disease markers (CRP $p=0.718$, ESR $\mathrm{p}=0.179$, RF titre $\mathrm{p}=0.466$, ACPA titre $\mathrm{p}=0.712$ ). In contrast, analyzing TRAF1 expression in different types of blood cells, significantly higher levels of TRAF1 mRNA were detected in monocytes carrying the AA genotype compared with the GG genotype at rs3761847, both in unstimulated (2.26-fold) as well as LPS+IFN $\gamma$ activated cells (2.04-fold; $p<0.05, n=3$ each). No significant association of TRAF1 expression or transcript variant utilization with rs3761847 was observed in the other blood-derived cell types studied, i.e. PBMC, macrophages and neutrophils, 
or using other stimuli, i.e. IL-4 and TNF- $\alpha$, indicating a specific function for the rs3761847 polymorphism in unstimulated and LPS+IFN $\gamma$-activated monocytes. Conclusions: Our findings suggest that there is no relationship between invasive capacity of RASF or expression of TRAF1-C5 genes and genotype at rs3761847. In contrast, we report an association of the rs3761847 genotype and TRAF1 expression in monocytes. These data underline the importance of studying genotype-phenotype associations in the different cell types relevant for RA pathogenesis.

Disclosure of Interest: None declared

DOI: 10.1136/annrheumdis-2017-eular.6066

\section{THU0015 INVESTIGATION OF JUVENILE IDIOPATHIC ARTHRITIS (JIA) IN GREECE: NEW SUSCEPTIBILITY LOCI}

M.I. Zervou ${ }^{1}$, D.G. Dimopoulou ${ }^{2}$, E. Eliopoulos ${ }^{3}$, M. Trachana ${ }^{4}$

P. Pratsidou-Gkertsi ${ }^{4}$, A. Andreou ${ }^{3}$, A. Garyfallos ${ }^{2}$, G.N. Goulielmos ${ }^{1} .{ }^{1}$ Medical School, University of Crete, Heraklion, Crete, ${ }^{2} 4$ th Department of Internal Medicine, Hippocratio Hospital, Aristotle University of Thessaloniki, Thessaloniki; ${ }^{3}$ Biotechnology, Agricultural University of Crete, Athens; ${ }^{4} 1$ st Department of Pediatrics, Aristotle University of Thessaloniki, Thessaloniki, Greece

Background: Juvenile idiopathic arthritis (JIA) is an autoimmune disease characterized by persistent chronic arthritis, in which both genetic and environmental components are involved [1]. Different genetic variations have been reported as risk factors for JIA, but a difficulty of the replication of results in different ethnic backgrounds indicates the existence of an ethnic heterogeneity of genetic factors for JIA.

Objectives: We sought to validate three single nucleotide polymorphisms (SNPs), namely PTPRC (rs10919563), TYK2 (rs34536443) and PRKCQ (rs4750316), previously found to be associated with JIA [2-4], and to investigate whether the 27-bp VNTR polymorphism on intron 4 of eNOS, which is associated with various autoimmune diseases so far [5], is associated with risk for JIA in Greece.

Methods: The sample set consisted of $125 \mathrm{JIA}$ patients and 221 healthy controls from Northern Greece. Genotyping of the three SNPs was performed with Taqman primer-probe sets, using a Real-Time PCR platform (Applied Biosystems, ViiA ${ }^{\mathrm{TM}}$ 7 Real-Time PCR System), while eNOS VNTR polymorphism was genotyped by PCR. Odds ratios (OR) and $95 \%$ confidence intervals $(\mathrm{Cl})$ were calculated and the statistical difference in allele distribution was assessed by means of $x^{2}$ test or Fisher's exact test. Bioinformatic analysis was performed using BlastP, Pymol and Maestro and Desmond (Schrodinger Inc.).

Results: A case-control association study was conducted enrolling 4 successfully genotyped markers. eNOS only was found to be associated with JIA. Genotype $\mathrm{a} / \mathrm{a}$ and allele "a" were more common in individuals with JIA than in controls $(p<0.0001, O R=0.15,95 \% \mathrm{Cl} 0.065-0.37$ and $p<0.0001, \mathrm{OR}=0.34,95 \% \mathrm{Cl}$ $0.23-0.49$, respectively). No associations with JIA were detected for TYK2, $P T P R C$ or $P R K C Q$. Aiming to investigate the structural consequences and the structure/function relationships accompanying the Pro1104 to Ala (rs34536443) mutation on TYK2 protein, bioinformatics analysis was performed. Combining 3D-modeling and Molecular Dynamics simulations we have noted changes in structural flexibility, affecting the functionality of the kinase domain of TYK2.

Conclusions: This study demonstrated for the first time that eNOS VNTR polymorphism is associated with susceptibility to JIA, thus suggesting that the risk allele "a" may confer susceptibility to clinically distinct disorders. Apart from the previously reported evidence for the role of PTPRC rs10919563, PRKCQ rs4750316 and TYK2 rs34536443 in an increased risk for JIA, our results demonstrate no association of these genes with JIA in the Greek population. However, the lack of association of PTPRC SNP with JIA is in line with previous data reported from cohorts in US and Australia. Taken together, the results highlight the importance of comparative studies in different populations, considering that replication of previously identified markers is paramount to determine which SNPs represent true risk loci, thus pointing towards key disease pathways which warrant further study.

References:

[1] Ravelli and Martini (2007). Lancet 369:767-78.

[2] Hinks et al (2010). Ann Rheum Dis 69:1049-53.

[3] Hinks et al. (2012). Ann Rheum Dis 71:1117-21.

[4] Hinks et al (2013). Nat Genet 45: 664-9.

[5] Vazgiourakis et al (2007). Lupus 16:867-74.

Disclosure of Interest: None declared

DOI: 10.1136/annrheumdis-2017-eular.2258

\section{THU0016 A COMPREHENSIVE CONTRIBUTION OF GENES OF THE HYPOXIA INDUCIBLE FACTOR-1 ALPHA SIGNALING PATHWAY TO KNEE OSTEOARTHRITIS SUSCEPTIBILITY}

J. Fernandez-Torres $^{1,2}$, G.A. Martínez-Nava ${ }^{1}$, A. López-Reyes ${ }^{1}$,

Y. Zamudio-Cuevas ${ }^{1}$, D. Clavijo-Cornejo ${ }^{1}$, K. Martínez-Flores ${ }^{1}$,

M.C. Gutiérrez-Ruíz ${ }^{2}$, L.E. Gómez-Quiroz ${ }^{2}$, C. Pineda ${ }^{1} .{ }^{1}$ Laboratorio de Líquido Sinovial, Instituto Nacional de Rehabilitacion; ${ }^{2}$ Cellular Physiology Laboratory, Universidad Autónoma Metropolitana-Iztapalapa, Mexico City, Mexico

Background: The hallmark of osteoarthritis (OA) is the breakdown of articular cartilage. Articular cartilage is an avascular tissue, and this generates a hypoxic microenvironment. Hypoxia inducible factor $-1 \alpha(\mathrm{HIF}-1 \alpha)$ is the main transcriptional regulator of cellular and developmental response to hypoxia.

Objectives: The present study was designed to investigate whether genetic polymorphisms of the HIF-1 $\alpha$ signaling pathway are involved in the development of knee OA.

Methods: A total of 243 unrelated Mexican-mestizo individuals comprising 93 knee OA patients and 150 healthy controls were recruited into the study. 42 genetic polymorphisms from 22 genes involved in the HIF-1 $\alpha$ signaling pathway (PIK3R1, AKT2, GSK3B, IL6, AGER, HIF1A, EGLN1, VHL, HIF1AN, VEGFA, EPO, NOS2, NOS3, IGF1, EGF, EDN1, MMP1, MMP3, MMP13, CA, COL2A1, COL3A1) were genotyped in cases and controls using TaqMan-based allelic discrimination assays.

Results: After adjusting for age, sex and admixture, significant associations with knee OA were found for 7 SNPs in the case-control study. The following genotypes and alleles were associated with protection against $\mathrm{OA}$ : the CT genotype of the HIF1AN rs 11190613 polymorphism (OR=0.44, 95\% $\mathrm{Cl}=0.19-1.0$, $P=0.05$ ); the AA genotype of the VEGFA rs 1570360 polymorphism (OR $=0.14$, $95 \% \mathrm{Cl}=0.02-0.69, P=0.016)$; the GT genotype and T allele of the VEGFA rs729761 polymorphism $(\mathrm{OR}=0.47,95 \% \mathrm{Cl}=0.22-1.0, P=0.05$; and $\mathrm{OR}=0.51$, $95 \% \mathrm{Cl}=0.27-0.97, P=0.041$, respectively); the GA genotype of the COL2A1 rs 1793953 polymorphism (OR=0.40, 95\% $\mathrm{Cl}=0.20-0.79, P=0.008)$; and the $\mathrm{GG}$ genotype and $\mathrm{G}$ allele of the CKM rs 4884 polymorphism $(\mathrm{OR}=0.34,95 \% \mathrm{Cl}=0.14-$ $0.84, P=0.019$; and $\mathrm{OR}=0.51,95 \% \mathrm{Cl}=0.32-0.82$, respectively). Otherwise, the CT genotype of the COL3A1 rs2138533 polymorphism (OR=2.89, 95\% $\mathrm{Cl}=1.28-6.5$, $P=0.01$ ); and the GA genotype of the IGF1 rs35767 polymorphism (OR=2.22, 95\% $\mathrm{Cl}=1.11-4.43, P=0.024$ ) were associated with an increased risk of OA. However, by using of epistatic interactions between HIF-1 $\alpha$ pathway polymorphisms, we found that the gene-gene interaction had a synergistic effect over the estimated OR-values (see table).

\begin{tabular}{|c|c|c|c|c|}
\hline a & $\mathrm{b}$ & $\mathrm{OR}_{\mathrm{i}}$ & $\mathrm{OR}_{\mathrm{c}}{ }^{*}$ & $P_{\mathrm{int}}$ \\
\hline VEGFA rs 1570360 & COL3A1 rs2138533 & & & \\
\hline \multirow[t]{3}{*}{ GG } & $\mathrm{CC}$ & 1 & & 0.027 \\
\hline & CT & 2.89 & 4.51 & \\
\hline & TT & 1.24 & 11.1 & \\
\hline COL3A1 rs2138533 & IGF1 rs35767 & & & \\
\hline \multirow[t]{2}{*}{$\mathrm{T}$} & G & 1 & & 0.037 \\
\hline & A & 1.49 & 3.26 & \\
\hline CKM rs 4884 & COL3A1 rs2138533 & & & \\
\hline \multirow[t]{2}{*}{ A } & $\mathrm{C}$ & 1 & & 0.036 \\
\hline & $\mathrm{T}$ & 1.27 & 4.08 & \\
\hline COL2A1 rs1793953 & HIF1AN rs11190613 & & & \\
\hline \multirow[t]{2}{*}{ A } & $\mathrm{T}$ & 1 & & 0.05 \\
\hline & C & 0.71 & 0.35 & \\
\hline
\end{tabular}

$\mathrm{OR}_{\mathrm{i}}=$ initial OR-value; $\mathrm{OR}_{\mathrm{c}}{ }^{*}=$ combined OR-value obtained by "b" column interaction with "a" column; $P_{\text {int }}=P$-value of the intreraction.

Conclusions: In this study we could observe that the gene-gene interaction of the HIF-1 $\alpha$ signaling pathway highly increases the risk of developing $O A$, with the exception of COL2A1 and HIF1AN interaction which had a protective role against $O A$. Further studies are needed to validate this results.

References:

[1] Fernández-Torres J, et al. Polymorphic variation of hypoxia inducible factor-1 A (HIF1A) gene might contribute to the development of knee osteoarthritis: a pilot study. BMC Musculoskelet Disord 2015; 16:218.

[2] Rodríguez-Fontanela $\mathrm{C}$, et al. Assessment of osteoarthritis candidate genes in a meta-analysis of nine genome-wide association studies. Arthritis Rheumatol 2014; 66:940-949.

Disclosure of Interest: None declared

DOI: 10.1136/annrheumdis-2017-eular.2131

\section{THU0017 COMBINATION OF EGFR AND BLYS GENE EXPRESSION IN LUPUS NEPHRITIS}

L.A. Mas ${ }^{1,2}$, S. Retamozo ${ }^{2,3}$, F. Bonisconti ${ }^{1,2}$, E.V. Palomino ${ }^{1,2}$, J.P. Pirola ${ }^{2,3}$, V. Saurit $^{2,3}$, A. Alvarellos ${ }^{2,3}$, T. Alvarellos ${ }^{1,2}{ }^{1}$ Immunogenetics, Hospital Privado. Universitario de Córdoba; ${ }^{2}$ Instituto Universitario de Ciencias Biomédicas de Córdoba; ${ }^{3}$ Rheumatology, Hospital Privado. Universitario de Córdoba, Cordoba, Argentina

Background: Lupus nephritis (LN) is a severe complication of Systemic Lupus Erythematosus (SLE). Non-invasive biomarkers are needed for diagnosis of LN and to identify patients at risk of a renal flare (1). Thus the presence of biomarkers associated with inflammation, tissue damage or cell activation in the urine of patients with $L N$ may be a useful tool in the evaluation of LN patients.

The glomerular filtration rate (GFR) is considered the best overall index of renal function in health and disease. Because GFR is difficult to measure in clinical practice, most clinicians estimate the GFR (eGFR) from the serum creatinine concentration (2).

B Lymphocyte Stimulator (BLyS) is a cytokine that fosters B cell activation, antibody production, B cell - T cell interaction and plasma cell survival. These events have been demonstrated to play a role in patients with LN (3).

Objectives: We evaluated urinary levels of BLyS as biomarker for LN and their relationship with eGFR. 